\title{
28 Research Square \\ Validity Of Self-reported Alcohol And Other Drug Use Among Pregnant Women Attending Midwife Obstetric Units In The Cape Metropole, South Africa
}

Petal Petersen Williams ( $\sim$ petal.petersen@mrc.ac.za)

South African Medical Research Council https://orcid.org/0000-0001-5535-2458

Catherine Mathews

South African Medical Research Council

Esme Jordaan

South African Medical Research Council

Yukiko Washio

RTI International

Mishka Terplan

Virginia Commonwealth University Medical Center

Charles Parry

South African Medical Research Council

Research article

Keywords: Validity, ASSIST, Pregnancy, Substance Use, South Africa

Posted Date: August 22nd, 2019

DOl: https://doi.org/10.21203/rs.2.13377/v1

License: (9) (i) This work is licensed under a Creative Commons Attribution 4.0 International License.

Read Full License 


\section{Abstract}

Background: This paper examines the validity of self-report of alcohol and other drug use in a South African cohort of pregnant women by comparing simple questioning about alcohol and other drug use (yes/no), a standardized screening tool (Alcohol, Smoking and Substance Involvement Screening Test; ASSIST, and urine analysis. Method: An observational study among women in eleven public prenatal clinics was conducted. Dichotomous verbal screening is a standard of care, and pregnant patients reporting alcohol and substance use in dichotomous verbal screenings were asked to engage in screening using the ASSIST and urinalysis. Results: Significant agreements between dichotomous and ASSIST scores were observed $(K=0.73-0.76)$. A higher rate of self-reported $(36.9 \%)$ alcohol use was detected, relative to urine screening (19.6\%) with a predictive value of 34.9; while underreporting of illicit substance use was observed ( $3.6 \%$ self-report vs $8.8 \%$ urine screening) with an overall predictive value of 50.0. Conclusion: Dichotomous verbal screening was considered valid after comparison with the ASSIST; however, combined use with urine screenings can be recommended especially for identifying illicit substance use in order to accurately detect alcohol and other drug use in pregnancy so that women can be identified and referred for appropriate interventions where needed.

\section{Background}

Prenatal substance use (illicit substances, alcohol, and tobacco) is one of the largest modifiable risk factors for significant gestational complications for both women and their newborns $[1,2,3,4]$.

Polysubstance use further increases the risk for adverse pregnancy outcomes including miscarriage, low birth weight, preterm birth, stillbirth, and neurocognitive disorders [1, 2, 3, 4, 5]. According to self-reported maternal smoking, alcohol use, and drug use each increased the likelihood of having neonatal morbidity including impaired Apgar scores ( $<7$ at 5-min postpartum), neonatal resuscitation, and neonatal intensive care unit (NICU) admission [6]. While studies among certain populations of pregnant women attending antenatal services in the Western Cape have reported on rates of substance use $[7,8,9]$, to date, national prevalence of prenatal substance and alcohol use is not known in South Africa, unlike in the U.S. [10]. However, locally, the prevalence data of substance and alcohol use exist for overall populations $[11,12]$.

Discrepancies between self-reported rate of substance and alcohol use and biochemically verified use have been well documented, in which self-reported substance and alcohol use generally demonstrated low sensitivity relative to biomarkers $[13,14,15]$, in part due to fear of judgment [16]. Nevertheless, biochemical screenings do not necessarily demonstrate clinical efficacy nor may they be ethically acceptable due to the legal implications of prenatal substance use, at least in the U.S. [17]. Although a severely punitive approach such as loss of child custody does not happen in South Africa [18], fear of exposure to stigmatization by healthcare providers may increase under-reported substance and alcohol use among pregnant women when asked in healthcare settings [19]. A combined use of biochemical screenings with a standard self-report instrument (such as the Alcohol Smoking and Substance Involvement Screening Test; ASSIST) is encouraged for identifying substance use in pregnant women [17]; however, little international empirical evidence supports this notion so far. 
The ASSIST is a brief screening questionnaire consisting of 8 items and was developed by the WHO and an international team of substance use researchers as a simple method of screening for hazardous, harmful and dependent use of alcohol, tobacco and other psychoactive substances. The ASSIST was designed to screen for substance abuse in primary care settings and pregnant women have been recommended as a target group suitable for an ASSIST screening program by the developers of this tool [20]. A validity study conducted with 1047 subjects from seven countries, including Australia, Brazil, India, Thailand, the UK, USA, and Zimbabwe, showed that the ASSIST had good concurrent, construct, predictive and discriminative validity [21]. The current study examined to what extent a self-report screen can adequately identify prenatal alcohol and other drug (AOD) use with a South African cohort attending prenatal care.

\section{Methods}

Ethical approval to conduct the research was obtained from the Faculty of Health Sciences Research Ethics Committee at the University of Cape Town. Permission to conduct the survey in the MOUs was obtained from the Western Cape Department of Health.

\section{Study design and setting}

A two-stage cluster survey design was used. The study population comprised pregnant women 16 years or older, presenting for their first antenatal visit at one of 11 public, community-based clinics called Midwife Obstetric Units (MOUs), scattered across eight health sub-districts, for antenatal care and delivery in the greater Cape Town, a city in the Western Cape region of South Africa. MOUs are birthing units run by midwives in the community for primary health care patients seeking antenatal care and/or delivery. Midwives at these facilities handle deliveries and referral to a hospital can occur at any stage, should this be necessary.

Stage 1 screened all pregnant women attending the clinic for their first prenatal visit in order to determine eligibility for participation and stratify women into one of three groups (Stratum A comprised woman who reported drug use; Stratum B comprised woman who reported alcohol use only; and Stratum C comprised women who reported no AOD use). Proportional sampling allocation was used across the $11 \mathrm{MOUs}$, using the total clinic attendance by pregnant women recorded by the Department of Health one year prior, to determine the number of first bookings from each MOU to be screened at stage 1. A second stage subsample was selected from the first stage group of pregnant women, for detailed interviewing about AOD use and for urine testing. The second stage selection of women from the MOUs was done with unequal probability sampling as follows. Since it was important to ensure an adequate sample size for the positive drug group, all women belonging to stratum $A$ were selected. For the presumed larger stratums $B$ and $\mathrm{C}$, systematic random sampling techniques were employed. It was planned that from the alcohol only stratum, every 10 th observation and from the no AOD use stratum every 5th observation was to be sampled. The choices of 10 th and 5th observation were arbitrary, although we believed that a certain measure of underreporting would take place and reduce the no AOD group, and hence a larger proportion 
of women from group $C$ than group $B$ were being recruited. The second stage sampling and subsequent interview and urine collection occurred immediately. We selected a 2-stage design because it was not economically feasible to do urine tests for six drugs and alcohol on all the women from Stage 1 and sample size calculations indicated sufficient power to do so.

\section{Measures of substance use}

Three assessments of alcohol and other drug use were used to estimate prevalence. 1) Dichotomous selfreport where women were asked about alcohol or drug use during their current pregnancy or in the three months before they knew they were pregnant. These questions resemble current standard care during intake where pregnant women are asked direct questions about current tobacco, alcohol and drug use, 2) a validated assessment based on the Alcohol, Smoking and Substance Involvement Screening Test (ASSIST), and 3) urine screening.

\section{Study procedure}

The study was conducted over a period of approximately 100 days from February - March 2010 and again from November 2010 - March 2011. This split occurred in all clinics except one where data collection was completed during the first period. Fieldwork had to be suspended for seven months due to unforeseen requirements from the funder to have projects reviewed again, resulting in the split between the two data collection periods. To be eligible for participation in this survey, women had to be pregnant, attending the clinic for their first/booking visit, be 16 years or older, and give written consent to participate in the study and provide a urine sample to be tested for AOD. After completion of the stage 2 interview, each participant received reimbursement for their time and participation in the form of a chain store voucher to the value of R50 (approximately $\$ 6$ ). They were also provided with a resource list.

\section{Data Analysis}

Appropriate sampling weights were determined according to the study design to generalize the results to the described population of pregnant women. In total, 5231 pregnant women were screened to assess self-reported prevalence estimates. Of these, 684 (13.1\%) were intentionally sub-sampled and completed an interviewer-administered questionnaire and provided a urine sample for biological screening. Selfreported alcohol and drug use was compared with urine screens. A survey analysis was conducted, using appropriate weights for the proportional allocation of women, to estimate the reported drug and alcohol prevalence and $95 \%$ confidence limits in the screening sample. A finite population correction was also used. Prevalence was estimated for the drug group, the alcohol only group and the no AOD group. The prevalence for the larger group of women using alcohol regardless of drug use was also estimated.

For estimating the prevalence reported using the ASSIST and the urinalysis involving the sub-sample, a survey analysis was used, incorporating appropriate weights for the women belonging to the three strata $A, B$ and $C$. The proportional clinic weights and the sub-sample weights were combined to obtain the 
appropriate sampling weights for calculating the prevalence of drug and alcohol use, with standard errors and $95 \%$ confidence limits in the sub-sample.

First, agreement between the self-reported substance use using simple, dichotomous questions (yes/no) and the substance use measured indirectly using the ASSIST relating to the last 3 month's substance use (aggregated responses; yes=once or twice, monthly, weekly, daily; no=never or not in the past 3 months) was measured by the kappa statistic and $95 \%$ confidence interval. The kappa values were calculated on the weighted values in the $2 \times 2$ tables. To indicate the strength of the agreement the standards of Landis and Koch were used: $<0.00=$ poor; $0.00-0.20=$ slight; $0.21-0.40=$ fair; $0.41-0.60=$ moderate; $0.61-0.80=$ substantial; and 0.81-1.00 = almost perfect [22].

Second, agreement between the responses to the ASSIST and urinalysis results were examined, looking at the sensitivity, specificity, positive predicted value (PPV), and the negative predicted value (NPV) with $95 \%$ confidence intervals were reported as measures of validity. The analyses were undertaken for cannabis, heroin, methamphetamine, and benzodiazepine as well as for all drugs combined. For the urinalysis, the standardised cut-off values for the individual drugs were used to indicate positive use of the specific drug. The observed frequency tables were reported and thereafter the $2 \times 2$ tables. The measures of validity were calculated for the weighted values in the $2 \times 2$ tables. Analyses were conducted using SAS version 9.4.

\section{Results}

\section{Characteristics of the sample}

In total 5231 pregnant women screened for AOD use during their current pregnancy and/or in the three months before they knew they were pregnant. A total of 684 patients received ASSIST screenings (Refer to Figure 1 for a detailed sampling flowchart).

The 684 participants had a mean age of 26 years (SE=0.25); 60.3\% were Black African, $39.1 \%$ Coloured, and $1.6 \%$ White or Asian. Ninety-two percent had some secondary education, $66.9 \%$ were never married/single and $56.9 \%$ unemployed. The majority of participants had five or more of the listed assets in their homes (75.9\%) indicating higher socio-economic status (SES) compared to those with four or less items. Approximately $70 \%$ were multiparous. Over half the participants reported that their current pregnancy was unplanned (68.2\%), and the mean gestational age at the booking visit was 18.9 weeks with the majority of women booking in their second trimester (57.4\%) (Table 1$)$.

\section{Prevalence of alcohol and other drug (AOD) use}

An estimation of the prevalence of substance use was conducted taking into account stratified proportional allocation, adjusting variance for stratified proportional sampling. Three assessments of drug use were used to estimate prevalence: dichotomous self-report in the screened sample ( $N=5231)$; an assessment based on the Alcohol, Smoking and Substance Involvement Screening Test (ASSIST) 
$(\mathrm{N}=684)$; and biological urine tests $(\mathrm{N}=592)$. Ninety-five urine samples were not tested for any $(\mathrm{n}=34)$ or some $(n=61)$ substances because of a fault of the testing laboratory, which accounted for $12.2 \%$ of the total samples.

Dichotomous self-report $(\mathrm{N}=5231)$ showed that 3.6\% (95\% Cl: 3.1-4.0) reported at least one illicit substance use, and $36.9 \%$ (95\% Cl: 35.6-38.1) reported alcohol use. ASSIST screening ( $\mathrm{N}=684)$ showed that $4.7 \%$ reported at least one illicit substance use, and $36.8 \%$ reported alcohol use. Urinalyses $(\mathrm{N}=592)$ showed that $8.8 \%$ (95\% Cl: 6.7-10.9) tested positive for at least one illicit substance use, and 19.6\% (95\% Cl: 16.3-22.8) tested positive for alcohol use.

\section{Agreement between dichotomous and ASSIST screenings}

The overall measure of agreement between the dichotomous self-reported AOD rate and AOD rate screened by ASSIST was statistically significant $(\mathrm{K}=0.76 ; 95 \% \mathrm{Cl} 0.71-0.81$ and $\mathrm{K}=0.73 ; 95 \% \mathrm{Cl} 0.59-0.86$ for alcohol and drug use respectively), indicating a strong agreement between the two means of verbal screenings.

\section{Agreement between dichotomous and urinalyses}

Urinalyses showed the rate of illicit substance use that was higher than dichotomous self-report (3.6\% vs. $8.8 \%)$, and the rate of alcohol use that was lower than dichotomous self-report (36.9\% vs. $19.6 \%)$.

\section{Agreement between ASSIST and urine screenings}

Table 2 shows agreements between ASSIST and urine screening with respect to sensitivity, specificity, and predictability. The weighted sensitivity (the proportion of self-reports of alcohol use among those testing positive for alcohol use) of self-report for alcohol use using the ASSIST as compared to biomarkers was $58.5 \%$ (Cl: 48.9-68.1), and the weighted specificity (the proportion of self-reports of no alcohol use among those testing negative for alcohol use) was $68.7 \%$ (Cl: 63.9-73.6). The positive predictive value, which indicates how likely someone with a positive test result is to actually have the characteristic or be a user of alcohol, was $34.9 \%$.

The weighted sensitivity of self-report for other drug use was $22.5 \%$, and the specificity was $97.5 \%$. The positive predictive value (PPV) was $50.0 \%$. The weighted sensitivity for self-report of Cannabis use compared to biomarkers was $51.4 \%$ (95\% Cl: $27.8-74.9)$, and the specificity was $98.4 \%$ (95\%Cl: $97.7-99.2)$ with the PPV of $37.6 \%$. Sensitivity analysis was not conducted for heroin use due to the small sample size $(n=5)$ with specificity of $99.9 \%$. The weighted sensitivity of self-report of methamphetamine was $13.3 \%$, and the specificity was $98.5 \%$ with the PPV of $46.8 \%$. No participant reported benzodiazepine use (Table 2).

\section{Discussion}


The current study showed that ASSIST verbal screening showed a good agreement with dichotomous verbal screening; however, ASSIST identified more alcohol use and less illicit substance use compared to urine screenings. This indicates that pregnant women in the Western Cape region of South Africa are willing to disclose alcohol use; however tend to underreport substance use.

Data from studies conducted in the USA show that pregnant women are more likely to underreport alcohol use during pregnancy for the concern of stigmatization [16]. Although public health messaging regarding harmful effects of drinking during pregnancy is available, and a brief intervention to educate pregnant mothers has been effective with the South African cohort [23], socioeconomically disadvantaged pregnant women enter late in prenatal care as found in this study. The reasons for this are complex and varies but includes importantly women's perceptions of quality of care, which influences their health seeking practices [24]. It should be noted that underreporting of prenatal alcohol use might increase as the norm around perceived harm of prenatal alcohol use changes.

Contrary to reporting alcohol use, report of illicit substance use or noncompliance with a treatment plan among mothers can result in rather severe legal consequences such as loss of child custody and incarceration in the U.S [17]. This is not the case with South Africa to date [18], and potential consequence of reported illicit substance use is assignment of case management by a social worker; however, stigmatization by healthcare professionals on prenatal illicit substance use [19] is potentially the reason of underreported illicit substance use in the current study. Due to low disclosure rate of illicit substance use among pregnant women, the combined verbal and urine screenings are recommended to determine the national prevalence of prenatal substance use; while, verbal screening only captures the prevalence of prenatal alcohol use. However, questions around the affordability of this remain and the availability of resources to respond appropriately.

A few limitations are noted in the current study. Not all pregnant patients volunteered for the study; therefore, this was considered a convenient sample. Not all participants who were screened with ASSIST had their urines tested due to laboratory issues with some samples being inadvertently destroyed.

The current study contributes to the existing literature in the following ways: (1) ASSIST verbal screening demonstrated a high agreement with dichotomous verbal screening with a South African cohort which implies we can continue using simple questioning about use (yes/no); (2) the rate of selfreported prenatal alcohol use was higher than that of urine screening with the cohort, contrary to the U.S.; (3) the rate of self-reported illicit substance use was lower than that of urine screening with the cohort, consistent with the literature in the U.S.

In summary, the current study examined agreements between dichotomous verbal screening, validated verbal screening, and urine screening in prenatal alcohol and substance use with a South African cohort. High rate of self-reported prenatal alcohol use was observed; however, illicit substance use was under reported. Dichotomous verbal screening was considered valid after comparison with the validated verbal screening method; however, combined use with urine screenings can be recommended 
especially for identifying illicit substance use in order to accurately detect alcohol and other drug use in pregnancy so that women can be identified and referred for appropriate interventions where needed.

\section{Declarations}

Ethics approval and consent to participate

Ethical approval to conduct the research was obtained from the Faculty of Health Sciences Research Ethics Committee at the University of Cape Town. Permission to conduct the survey in the MOUs was obtained from the Western Cape Department of Health.

\section{Consent for publication}

Not applicable

\section{Availability of data and material}

The datasets used and/or analysed during the current study are available from the corresponding author on reasonable request.

\section{Competing interests}

The authors declare that they have no competing interests.

\section{Funding}

The research was funded by the US President's Emergency Fund for AIDS Relief (PEPFAR) through the US Centers of Disease Control and Prevention (CDC) under the terms of 5U2GPS001137-05. Its contents are solely the responsibility of the authors and do not necessarily represent the official views of the CDC or PEPFAR. The CDC had no further role in the study design; in the collection, analysis and interpretation of data; in the writing of the report; or in the decision to submit the paper for publication. The research reported in this publication was also supported by the South African Medical Research Council. 
PPW designed the study and wrote the protocol. CP and CM made considerable contributions to the design of the study and assisted with the protocol development. EJ and PPW undertook the analysis. YW and MT provided expertise and guidance in addressing issues around validity in mapping out a manuscript. PPW and YW wrote the first draft of the manuscript. All authors contributed to and have approved the final manuscript.

\section{Acknowledgements}

The authors would like to acknowledge the support of our fieldwork staff, in particular Elmarie Nel and Nomtha Madlingozi, and the staff and patients at the various Midwife Obstetric Units (MOUs) where data were collected. The authors further wish to thank the Western Cape Provincial Department of Health for granting permission to conduct the survey in the antenatal facilities.

\section{References}

[1] Aliyu MH, Wilson RE, Zoorob R, Brown K, Alio AP, Clayton H, Salihu HM. Prenatal alcohol consumption and fetal growth restriction: potentiation effect by concomitant smoking. Nicotine Tobacco Research. 2009;11(1):36-43. doi:10.1093/ntr/ntn014.

[2] Bailey BA, McCook JG, Hodge A, McGrady L. Infant birth outcomes among substance using women: why quitting smoking during pregnancy is just as important as quitting illicit drug use. Maternal and Child Health Journal. 2012;16(2):414-422. doi:10.1007/s10995-011-0776-y.

[3] CDC. Alcohol use in pregnancy.

http://m.cdc.gov/en/HealthSafetyTopics/LifeStagesPopulations/Pregnancy/alcoholUsePublished 2013. Accessed June 19, 2017.

[4] Mark K, Desai A, Terplan M. Marijuana use and pregnancy: prevalence, associated characteristics, and birth outcomes. Arch Womens Ment Heal. 2015; doi:10.1007/s00737-015-0529-9.

[5] Quesada O, Gotman N, Howell HB, Funai EF, Rounsaville BJ, Yonkers KA. Prenatal hazardous substance use and adverse birth outcomes. J Matern Fetal Neonatal Med. 2012;25(8):1222-1227. doi:10.3109/14767058.2011.602143.

[6] Burstyn I, Kapur N, Cherry NM. Substance use of pregnant women and early neonatal morbidity: where to focus intervention? Canadian Journal of Public Health, 2010;101(2):149-153. http://www.ncbi.nlm.nih.gov/pubmed/20524381. Accessed August 17, 2015.

[7] Onah MN, Field S, van Heyningen T, Honikman S. Predictors of alcohol and other drug use among pregnant women in a peri-urban South African setting. International Journal of Mental Health Systems. 
[8] Petersen Williams P, Jordaan E, Mathews C, Lombard C, Parry CDH. Alcohol and Other Drug Use during Pregnancy among Women Attending Midwife Obstetric Units in the Cape Metropole, South Africa. Advances in Preventive Medicine. 2014, 10. doi: 10.1155/2014/871427.

[9] Vythilingum B, Roos A, Faure SC, Geerts L, Stein DJ. Risk factors for substance use in pregnant women in South Africa. South African Medical Journal = Suid-Afrikaanse Tydskrif Vir Geneeskunde. 2012;102(11 Pt 1), 851-854. doi: 10.7196/samj.5019

[10] SAMHSA. Results from the 2015 National Survey on Drug Use and Health: Detailed Tables, SAMHSA, CBHSQ. https://www.samhsa.gov/data/sites/default/files/NSDUH-DetTabs-2015/NSDUH-DetTabs2015/NSDUH-DetTabs-2015.htm. Published 2015. Accessed May 22, 2017.

[11] Peltzer K, Davids A, Njuho P. Alcohol use and problem drinking in South Africa: findings from a national population-based survey. Afr J Psychiatry. 2011;14(1):30-37.

http://www.ncbi.nlm.nih.gov/pubmed/21509408.

[12] Peltzer K, Ramlagan S, Johnson BD, Phaswana-Mafuya N. Illicit drug use and treatment in South Africa: a review. Subst Use Misuse. 2010;45(13):2221-2243. doi:10.3109/10826084.2010.481594.

[13] Bessa, M.A., Mitsuhiro, S.S., Chalem, E., Barros, M.M., Guinsburg, R., \& Laranjeira, R. (2010). Underreporting of use of cocaine and marijuana during the third trimester of gestation among pregnant adolescents. Addictive Behaviors, 35(3), 266-269. doi:10.1016/j.addbeh.2009.10.007.

[14] El Marroun, H., Tiemeier, H., Jaddoe, VWV, et al. Agreement between maternal cannabis use during pregnancy according to self-report and urinalysis in a population-based cohort: the Generation R Study. Eur Addict Res. 2011;17(1):37-43. doi:10.1159/000320550.

[15] Garg M, Garrison L, Leeman L, et al. Validity of Self-Reported Drug Use Information Among Pregnant Women. Matern Child Health J. 2016;20(1):41-47. doi:10.1007/s10995-015-1799-6.

[16] Muggli E, Cook B, O'Leary C, Forster D, Halliday J. Increasing accurate self-report in surveys of pregnancy alcohol use. Midwifery. 2015;31(3):e23-8. doi:10.1016/j.midw.2014.11.003.

[17] Terplan M, Minkoff H. Neonatal Abstinence Syndrome and Ethical Approaches to the Identification of Pregnant Women Who Use Drugs. Obstet Gynecol. 2017;129(1):164-167. doi:10.1097/AOG.0000000000001781.

[18] Gardner J. Should drinking during pregnancy be criminalised to prevent fetal alcohol spectrum disorder? South African J Bioeth Law. 2016;9(1):26. doi:10.7196/SAJBL.2016.v9i1.468.

[19] Petersen Williams P, Petersen Z, Sorsdahl K, Mathews C, Everett-Murphy K, Parry CDH. Screening and Brief Interventions for Alcohol and Other Drug Use Among Pregnant Women Attending Midwife Obstetric 
Units in Cape Town, South Africa: A Qualitative Study of the Views of Health Care Professionals. J Midwifery Womens Health. 60(4):401-409. doi:10.1111/jmwh.12328.

[20] Henry-Edwards S, Humeniuk R, Ali R, Poznyak V, Monteiro M. The Alcohol, Smoking and Substance Involvement Screening Test (ASSIST): Guidelines for Use in Primary Care. Geneva: World Health Organization; 2003.

[21] Humeniuk R, Ali R. Validation of the Alcohol, Smoking and Substance Involvement Screening Test (ASSIST) and Pilot Brief Intervention [Electronic Resource]: A Technical Report of Phase II Findings of the WHO ASSIST Project.; 2006. http://www.who.int/iris/handle/10665/43504.

[22] Landis RJ, Kock GG. The measurement of observer agreement for categorical data. Biometrics. 1977;33(1), 159-174. doi:10.2307/2529310.

[23] Rendall-Mkosi K, Morojele N, London L, Moodley S, Singh C, Girdler-Brown B. A randomized controlled trial of motivational interviewing to prevent risk for an alcohol-exposed pregnancy in the Western Cape, South Africa. Addiction. 2013;108(4):725-732. doi:10.1111/add.12081.

[24] Abrahams N, Jewkes R, Mvo Z. Health care-seeking practices of pregnant women and the role of the midwife in Cape Town, South Africa. Journal of Midwifery and Women's Health. 2001;46(4), 240-247.

\section{Tables}


Table 1: Participant demographic characteristics ( $\mathrm{n}=684)$

\begin{tabular}{|c|c|c|}
\hline & $\%$ & SE \\
\hline \multicolumn{3}{|l|}{ Age } \\
\hline $16-19$ & 14.7 & 1.48 \\
\hline $20-29$ & 62.9 & 2.08 \\
\hline $30-39$ & 19.5 & 1.73 \\
\hline $40-49$ & 2.8 & 0.73 \\
\hline \multicolumn{3}{|l|}{ Race } \\
\hline Black African & 60.3 & 1.70 \\
\hline Coloured & 39.1 & 1.71 \\
\hline White/Asian & 1.6 & 0.55 \\
\hline \multicolumn{3}{|l|}{ Education } \\
\hline Primary & 3.5 & 0.79 \\
\hline Secondary & 92.0 & 1.16 \\
\hline Tertiary - incomplete & 1.9 & 0.62 \\
\hline Tertiary - complete & 2.6 & 0.65 \\
\hline \multicolumn{3}{|l|}{ Marital Status } \\
\hline Legally married & 18.9 & 1.65 \\
\hline Traditionally married & 7.7 & 1.15 \\
\hline Living together & 4.1 & 0.84 \\
\hline Never married/single & 66.9 & 2.00 \\
\hline Divorced/separated/widowed & 2.4 & 0.68 \\
\hline \multicolumn{3}{|l|}{ Employment Status } \\
\hline Unemployed & 56.9 & 2.14 \\
\hline Employed full-time & 23.7 & 1.86 \\
\hline Employed part-time/self-employed & 12.0 & 1.43 \\
\hline Student & 7.4 & 1.10 \\
\hline \multicolumn{3}{|l|}{ Socio-economic status } \\
\hline Low ( 4 or less items) & 24.1 & 1.78 \\
\hline High (5 or more items) & 75.9 & 1.78 \\
\hline \multicolumn{3}{|l|}{ Parity } \\
\hline 0 & 34.0 & 2.00 \\
\hline 1 & 35.1 & 2.10 \\
\hline 2 & 19.6 & 1.70 \\
\hline 3 & 8.2 & 1.20 \\
\hline$\geq 4$ & 3.1 & 0.70 \\
\hline \multicolumn{3}{|l|}{ Planned Pregnancy } \\
\hline Yes & 31.8 & 2.10 \\
\hline No & 68.2 & 2.10 \\
\hline \multicolumn{3}{|l|}{ Gestational Age } \\
\hline $0-12$ weeks ( $1^{\text {st }}$ trimester) & 22.8 & 1.90 \\
\hline $13-24$ weeks (2 ${ }^{\text {nd }}$ trimester) & 57.4 & 2.30 \\
\hline $25-40$ weeks ( $3^{\text {rd }}$ trimester) & 19.8 & 1.80 \\
\hline
\end{tabular}

Table 2. Sensitivity, specificity, positive predictive value, and negative predictive value between ASSIST and urine screenings for alcohol and each substance 


\begin{tabular}{|c|c|c|c|c|c|c|c|c|}
\hline Substance & $\begin{array}{l}\text { Weighted } \\
\text { Sensitivity }\end{array}$ & $95 \% \mathrm{CI}$ & $\begin{array}{l}\text { Weighted } \\
\text { Specificity }\end{array}$ & $95 \% \mathrm{CI}$ & PPV & $\begin{array}{l}95 \% \\
\text { CI } \\
\end{array}$ & NPV & $\begin{array}{l}95 \% \\
\mathrm{CI} \\
\end{array}$ \\
\hline Alcohol & 58.5 & $\begin{array}{l}48.9- \\
68.1\end{array}$ & 68.7 & $\begin{array}{l}63.9- \\
73.6\end{array}$ & 34.9 & $\begin{array}{l}27.5- \\
42.2\end{array}$ & 85.3 & $\begin{array}{l}81.5- \\
89.0\end{array}$ \\
\hline Cannabis & 51.4 & $\begin{array}{l}27.8- \\
74.9\end{array}$ & 98.4 & $\begin{array}{l}97.7- \\
99.2\end{array}$ & 37.6 & $\begin{array}{l}21.0- \\
54.1\end{array}$ & 99.1 & $\begin{array}{l}98.4- \\
99.8\end{array}$ \\
\hline Heroin & NA & - & 99.9 & $\begin{array}{l}99.9- \\
100.0\end{array}$ & - & - & - & - \\
\hline Methamphetamine & 13.3 & $\begin{array}{l}8.5- \\
18.1\end{array}$ & 98.5 & $\begin{array}{l}97.5- \\
99.5\end{array}$ & 46.8 & $\begin{array}{l}29.9- \\
63.8\end{array}$ & 91.8 & $\begin{array}{l}89.3- \\
94.2\end{array}$ \\
\hline Benzodiazepine & NA & - & 99.9 & $\begin{array}{l}99.7- \\
99.9\end{array}$ & - & - & - & - \\
\hline Drugs overall & 22.5 & $\begin{array}{l}15.2- \\
29.9\end{array}$ & 97.5 & $\begin{array}{l}96.3- \\
98.7\end{array}$ & 50.0 & $\begin{array}{l}36.0- \\
64.0\end{array}$ & 91.9 & $\begin{array}{l}89.4- \\
94.3\end{array}$ \\
\hline
\end{tabular}

\section{Figures}



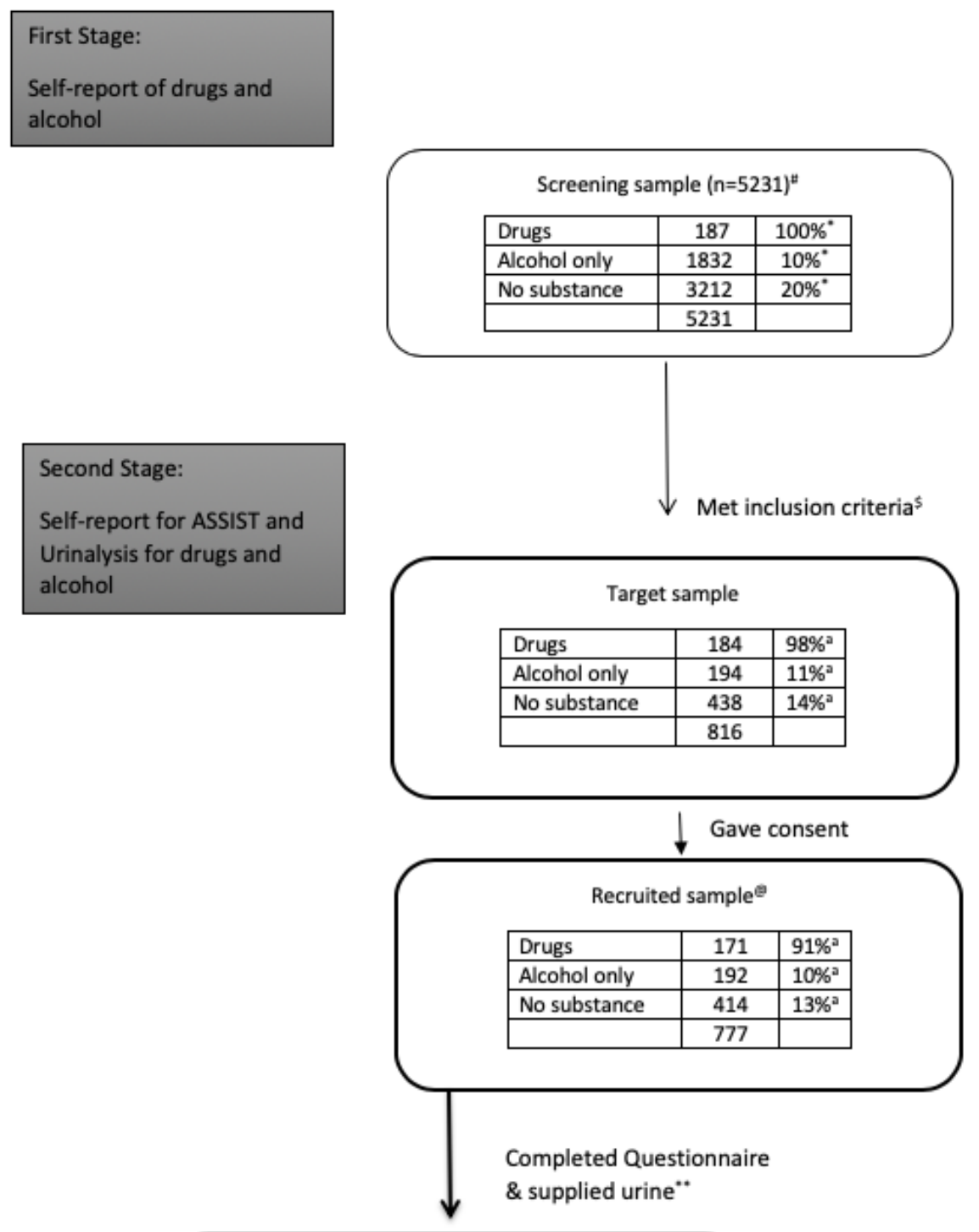

\section{Screening sample $(\mathrm{n}=5231)^{\sharp}$}

\begin{tabular}{|l|c|c|}
\hline Drugs & 187 & $100 \%^{\circ}$ \\
\hline Alcohol only & 1832 & $10 \%^{\circ}$ \\
\hline No substance & 3212 & $20 \%^{\circ}$ \\
\hline & 5231 & \\
\hline
\end{tabular}

\begin{tabular}{|l|c|c|}
\hline \multicolumn{3}{|c|}{ Final realized sample } \\
$\qquad$\begin{tabular}{|l|c|c|}
\hline Drugs & 155 & $83 \%^{\mathrm{ab}}$ \\
\hline Alcohol only & 169 & $9 \%^{\mathrm{ab}}$ \\
\hline No substance & 360 & $11 \%^{\mathrm{ab}}$ \\
\hline \multicolumn{3}{|l|}{} \\
\hline
\end{tabular} \\
\hline
\end{tabular}

" Proportional selection from 11 MOU clinics from a total population of 41715

"Intentional sampling proportions from each strata, these were changed during the study

pregnant, first booking, 16 years or older

"these women got an ID number

the denominator for percentages were the strata sizes from the screening sample

weights used in the analysis

"93 (12\%) women disappeared after recruitment for various reasons, this varies from $0 \%$ to $29 \%$ for the 11 clinics

\section{Figure 1}

Sampling flowchart. 